\title{
Learning and Inference in Mixed-State Conditionally Heteroskedastic Factor Models Using Viterbi Approximation
}

\author{
Mohamed Saidane ${ }^{1}$ and Christian Lavergne ${ }^{2}$ \\ 1 I3M, University Montpellier II, Place Eugene Bataillon, \\ CC - 05134095 Montpellier, France \\ saidane@math. univ-montp2.fr \\ http://www .math.univ-montp2.fr \\ 2 I3M, University Montpellier II, Place Eugene Bataillon, CC - 051 \\ 34095 Montpellier, France \\ Christian.Lavergne@math.univ-montp2.fr
}

\begin{abstract}
In this paper we develop a new approach within the framework of asset pricing models that incorporates two key features of the latent volatility: co-movement among conditionally heteroskedastic financial returns and switching between different unobservable regimes. By combining conditionally heteroskedastic factor models with hidden Markov chain models (HMM), we derive a dynamical local model for segmentation and prediction of multivariate conditionally heteroskedastic financial time series. The EM algorithm that we have developed for the maximum likelihood estimation, is based on a Viterbi approximation which yields inferences about the unobservable path of the common factors, their variances and the latent variable of the state process. Extensive Monte Carlo simulations and preliminary experiments obtained with a dataset on weekly average returns of closing spot prices for eight European currencies show promising results.
\end{abstract}

\section{Introduction}

The factor Model, also called Index Model, is one of the basic models in finance to analyze and describe the return generation process and the risk/reward relationships of a large number of assets. It has been used extensively in finance for measuring co-movement in and forecasting financial time series. Traditionally, these issues were considered in a static framework, but recently, the emphasis has shifted toward inter-temporal asset pricing models in which agents decisions are based on the distribution of returns conditional on the available information, which is obviously changing. Several researchers have used Factor-ARCH models to provide a plausible and parsimonious parameterization of the time varying covariance structure of asset returns. Engle et al. [1] apply such structures to study the dynamic behavior of the term structure of interest rates. Diebold and Nerlove 2] use a latent factor ARCH model to describe the dynamics of exchange 
rate volatility. Engle and Susmel [3] use the factor ARCH to test for common volatility in international equity markets.

A natural generalization of the different models proposed in the above literature to a multi-state model can be achieved by allowing for model transitions that are governed by a Markov chain on a set of possible models describing the different states of volatility. The originality of this work is the use of a piece-wise multivariate and linear process - which we also regard as a mixed-state dynamic linear system - for modeling the regime switches. In particular, we suppose that the observed series can be approximated using a time varying parameter model with the assumption that the evolution of these parameters is governed by a first-order hidden Markov process with $m$ states.

\section{Basic Model and Factor Structure}

The model that we propose supposes that excess returns depend both on unobservable factors that are common across the multivariate time series, and on unobservable different regimes that describe the different states of volatility. In this framework, we allow a dynamic structure for the conditional variances of the underlying factors in order to investigate possible time-varying latent processes, and their implications in modeling changes in covariance matrices over time. This new specification is defined by:

$$
\begin{gathered}
S_{t} \sim P\left(S_{t}=j / S_{t-1}=i\right) \\
t=1, \ldots, n \quad \text { and } \quad i, j=1, \ldots, m \\
\mathbf{f}_{s_{t}}=\mathbf{H}_{s_{t}}^{1 / 2} \mathbf{f}_{t}^{*} \quad \text { where } \quad \mathbf{f}_{t}^{*} \sim \mathcal{N}\left(\mathbf{0}, \mathbf{I}_{k}\right) \\
\mathbf{y}_{t}=\mathbf{X}_{s_{t}} \mathbf{f}_{s_{t}}+\varepsilon_{s_{t}} \text { with } \varepsilon_{s_{t}} \sim \mathcal{N}\left(\theta_{s_{t}}, \mathbf{\Psi}_{s_{t}}\right)
\end{gathered}
$$

where $S_{t} \sim P\left(S_{t}=j / S_{t-1}=i\right)$ is a hidden Markov chain indicating the state or the regime at the date $t$, and $\mathbf{y}_{t}$ is a $q \times 1$ random vector of observable variables (financial returns). In an unspecified state $S_{t}=j(j=1, \ldots, m), \theta_{j}$ are the $q \times 1$ mean vectors, $\mathbf{f}_{j t}$ the $k \times 1$ vectors of unobserved common factors, $\varepsilon_{j t}$ the $q \times 1$ vectors of idiosyncratic noises, $\mathbf{X}_{j}$ the $q \times k$ factor loadings matrices, with $q \geq k$ and $\operatorname{rank}\left(\mathbf{X}_{j}\right)=k, \boldsymbol{\Psi}_{j}$ are $q \times q$ diagonal and definite positive matrices of idiosyncratic variances, and $\mathbf{H}_{j t}$ the $k \times k$ diagonal and definite positive matrices whose elements are the variances of the common factors presumedly time varying and their parameters changes according to the regime. In particular, we suppose that the variances of the common factors follow switching $\operatorname{GQARCH}(1,1)$ processes, the $l$-th diagonal element of the matrix $\mathbf{H}_{j t}$ under an unspecified regime $S_{t}=j$ since $S_{t-1}=i$ being $h_{l t}^{(j)}=w_{j}^{l}+\gamma_{j}^{l} f_{l t-1}^{(i)}+\alpha_{j}^{l} f_{l t-1}^{(i) 2}+\delta_{j}^{l} h_{l t-1}^{(i)}$. 


\section{Viterbi Approximation for Latent Structure Inference}

The model developed above can be regarded as a random field with indices $i=1, \ldots, q, t=1, \ldots, n$ and $j=1, \ldots, m$. Therefore, it has a switching state-space representation, with $\mathbf{f}_{t}$ as the continuous state variables. The measurement and transition equations are, respectively, given by:

$$
\begin{gathered}
\mathbf{y}_{t}=\theta_{s_{t}}+\mathbf{X}_{s_{t}} \mathbf{f}_{s_{t}}+\varepsilon_{s_{t}} \\
\mathbf{f}_{s_{t}}=\mathbf{0} . \mathbf{f}_{s_{t-1}}+\mathbf{f}_{s_{t}}
\end{gathered}
$$

The task of Viterbi approximation approach is to find the best sequence of switching states $S_{t}$ and common factors $\mathbf{f}_{t}$ that minimizes the Hamiltonian cost in equation (1) for a given observation sequence $\mathcal{Y}_{1: n}$.

$$
\begin{gathered}
\mathcal{H}\left(\mathcal{F}_{1: n}, \mathcal{S}_{1: n}, \mathcal{Y}_{1: n}\right) \simeq \text { Constant }+\sum_{t=2}^{n} S_{t}^{\prime}(-\log \mathbf{P}) S_{t-1}+S_{1}^{\prime}(-\log \pi) \\
+\frac{1}{2} \sum_{t=1}^{n} \sum_{j=1}^{m}\left[\left(\mathbf{y}_{t}-\mathbf{X}_{j} \mathbf{f}_{j t}-\theta_{j}\right)^{\prime} \mathbf{\Psi}_{j}^{-1}\left(\mathbf{y}_{t}-\mathbf{X}_{j} \mathbf{f}_{j t}-\theta_{j}\right)+\log \left|\mathbf{\Psi}_{j}\right|\right] S_{t}(j) \\
+\frac{1}{2} \sum_{t=1}^{n} \sum_{j=1}^{m}\left[\mathbf{f}_{j t}^{\prime} \mathbf{H}_{j t}^{-1} \mathbf{f}_{j t}+\log \left|\mathbf{H}_{j t}\right|\right] S_{t}(j)
\end{gathered}
$$

where $\mathbf{P}$ and $\pi$ are, respectively, the HMM transition matrix and the vector of initial state probabilities.

Define first the "best" partial cost up to time $t$ of the measurement sequence $\mathcal{Y}_{1: t}$ when the switch is in state $j$ at time $t$ :

$$
J_{t, j}=\min _{\mathcal{S}_{1: t-1}, \mathcal{F}_{t}} \mathcal{H}\left[\mathcal{F}_{1: t},\left\{\mathcal{S}_{1: t-1}, S_{t}=j\right\}, \mathcal{Y}_{1: t}\right]
$$

Namely, this cost is the least cost over all possible sequences of switching states $\mathcal{S}_{1: t-1}$ and corresponding factor model states $\mathcal{F}_{1: t}$. In order to calculate this cost we first start by introducing some notation.

$$
\begin{aligned}
\mathbf{f}_{t / t-1}^{i(j)} & =\mathrm{E}\left[\mathbf{f}_{t} / \mathcal{Y}_{1: t-1}, S_{t}=j, S_{t-1}=i\right] \\
\mathbf{H}_{t / t-1}^{i(j)} & =\mathrm{E}\left[\left(\mathbf{f}_{t}-\mathbf{f}_{t / t-1}^{i(j)}\right)\left(\mathbf{f}_{t}-\mathbf{f}_{t / t-1}^{i(j)}\right)^{\prime} / \mathcal{Y}_{1: t-1}, S_{t}=j, S_{t-1}=i\right] \\
\mathbf{f}_{t / t}^{i(j)} & =\mathrm{E}\left[\mathbf{f}_{t} / \mathcal{Y}_{1: t}, S_{t}=j, S_{t-1}=i\right] \\
\mathbf{H}_{t / t}^{i(j)} & =\mathrm{E}\left[\left(\mathbf{f}_{t}-\mathbf{f}_{t / t}^{j}\right)\left(\mathbf{f}_{t}-\mathbf{f}_{t / t}^{j}\right)^{\prime} / \mathcal{Y}_{1: t}, S_{t}=j, S_{t-1}=i\right]
\end{aligned}
$$

From the theory of Kalman estimation it follows that for transition $i \rightarrow j$ the following time updates hold:

$$
\begin{gathered}
\mathbf{f}_{t / t-1}^{i(j)}=\mathbf{0 . \mathbf { f } _ { t - 1 / t - 1 } ^ { i }}=\mathbf{0} \quad \forall \quad i, j=1, \ldots, m \quad \text { and } \\
h_{l t / t-1}^{i(j)}=w_{l j}+\gamma_{l j} f_{l t-1 / t-1}^{i}+\alpha_{l j}\left[f_{l t-1 / t-1}^{i 2}+h_{l t-1 / t-1}^{i}\right]+\delta_{l j} h_{l t-1 / t-2}^{i}
\end{gathered}
$$


Given a new observation $\mathbf{y}_{t}$ at time $t$ each of these predicted estimates can now be filtered using Kalman measurement update framework:

$$
\begin{aligned}
\mathbf{f}_{t / t}^{i(j)} & =\mathbf{f}_{t / t-1}^{i(j)}+K_{t}(i, j) \mathbf{e}_{t}(i, j) \\
\mathbf{H}_{t / t}^{i(j)} & =\mathbf{H}_{t / t-1}^{i(j)}-K_{t}(i, j) \boldsymbol{\Sigma}_{t / t-1}^{i(j)} K_{t}(i, j)^{\prime}
\end{aligned}
$$

with $\mathbf{e}_{t}(i, j)=\mathbf{y}_{t}-\theta_{j}-\mathbf{X}_{j} \mathbf{f}_{t / t-1}^{i(j)} ; \boldsymbol{\Sigma}_{t / t-1}^{i(j)}=\mathbf{X}_{j} \mathbf{H}_{t / t-1}^{i(j)} \mathbf{X}_{j}^{\prime}+\mathbf{\Psi}_{j}$ and $K_{t}(i, j)=$ $\mathbf{H}_{t / t-1}^{i(j)} \mathbf{X}_{j}^{\prime} \Sigma_{t / t-1}^{i(j)-1}$. Each of these $i \rightarrow j$ transitions has a certain innovation cost $J_{t, t-1, i, j}$ associated with it, as defined in equation (7).

$$
J_{t, t-1, i, j}=\frac{1}{2} \mathbf{e}_{t}(i, j)^{\prime} \boldsymbol{\Sigma}_{t / t-1}^{i(j)-1} \mathbf{e}_{t}\left(i, j+\frac{1}{2} \log \left|\boldsymbol{\Sigma}_{t / t-1}^{i(j)}\right|-\log p_{i j}\right.
$$

one portion of this innovation cost reflects the continuous state transition, as indicated by the innovation terms in equation (5). The remaining $\operatorname{cost}\left(-\log p_{i j}\right)$ is due to switching from state $i$ to state $j$.

Obviously, for every current switching state $j$ there are $m$ possible previous switching states from which the system could have originated from. To minimize the overall cost at every time step $t$ and for every switching state $j$, one "best" previous state $i$ is selected:

$$
\begin{aligned}
J_{t, j} & =\min _{i}\left\{J_{t, t-1, i, j}+J_{t-1, i}\right\} \\
\delta_{t-1, j} & =\underset{i}{\arg \min }\left\{J_{t, t-1, i, j}+J_{t-1, i}\right\}
\end{aligned}
$$

the index of this state is kept in the state transition record $\delta_{t-1, j}$. Consequently, we now obtain a set of $m$ best filtered continuous states and their variances at time $t: \mathbf{f}_{t / t}^{j}=\mathbf{f}_{t / t}^{\delta_{t-1, j}(j)}$ and $\mathbf{H}_{t / t}^{j}=\mathbf{H}_{t / t}^{\delta_{t-1, j}(j)}$ with $h_{l t / t-1}^{j}=h_{l t / t-1}^{\delta_{t-1, j}(j)}$. Once all $n$ observations $\mathcal{Y}_{1: n}$ have been fused, the best overall cost is obtained as $J_{n}^{*}=\min _{j} J_{n, j}$. To decode the "best" switching state sequence, one uses the index of the best final state, $j_{n}^{*}=\arg \min J_{n, j}$, then traces back through $\delta_{t-1, j}$ : $j_{t}^{*}=\delta_{t, j_{t+1}^{*}}$.

The Switching model's sufficient statistics are now simply given by $\mathrm{E}\left(S_{t} /.\right)=$ $S_{t}\left(j^{*}\right)$ and $\mathrm{E}\left(S_{t} S_{t-1}^{\prime} /.\right)=S_{t}\left(j^{*}\right) S_{t-1}\left(j^{*}\right)^{\prime}$.1 Given the "best" switching state sequence, the sufficient factor model statistics can be easily obtained using the Rauch-Tung-Streiber smoothing (Rosti A-V.I and Gales M.J.F [4]). For example, $\mathrm{E}\left(\mathbf{f}_{t}, S_{t}(j) /.\right)=\mathbf{f}_{t / n}^{j_{t}^{*}}$ if $j=j_{t}^{*}$ and $\mathbf{0}$ otherwise.

\section{EM Algorithm}

The joint likelihood of the observations sequence $\mathcal{Y}_{1: n}$, the continuous state vector sequence $\mathcal{F}_{1: n}$ and the HMM state sequence $\mathcal{S}_{1: n}$ is given by:

\footnotetext{
${ }^{1}$ The operator $\mathrm{E}(/$.$) denotes conditional expectation with respect to the posterior$ distribution, e.g. $\mathrm{E}\left(\mathbf{f}_{t} /.\right)=\sum_{\mathcal{S}} \int_{\mathcal{F}} \mathbf{f}_{t} p(\mathcal{F}, \mathcal{S} / \mathcal{Y})$.
} 


$$
p(\mathcal{Y}, \mathcal{F}, \mathcal{S})=p\left(S_{1}\right) \prod_{t=2}^{n} p\left(S_{t} / S_{t-1}\right) \prod_{t=1}^{n} p\left(\mathbf{f}_{t} / S_{t}, \mathcal{D}_{1: t-1}\right) p\left(\mathbf{y}_{t} / \mathbf{f}_{t}, S_{t}, \mathcal{D}_{1: t-1}\right)
$$

where $\mathcal{D}_{1: t-1}=\left\{\mathcal{Y}_{1: t-1}, \mathcal{F}_{1: t-1}, \mathcal{S}_{1: t-1}\right\}$, is the information set at time $t-1$, $p\left(S_{1}\right)=\pi_{s_{1}}$ : the initial state probability and $p\left(S_{t} / S_{t-1}\right)=p_{s_{t-1} s_{t}}$ : the transition probabilities. In the first conditional maximisation step, the model parameters i.e. $\pi_{j}, p_{i j}, \mathbf{X}_{j}, \theta_{j}$ and $\mathbf{\Psi}_{j}$ can be obtained by maximizing the conditional expectation of this complete log-likelihood function.

In the second step, being given the new values above and the fact that $\mathbf{y}_{t} / \mathcal{Y}_{1: t-1}, S_{t}=j, \mathcal{S}_{1: t-1} \approx \mathcal{N}\left[\theta_{j}, \Sigma_{t / t-1}^{j}\right]$, the parameters $\phi_{j}=\left\{w_{j l}, \gamma_{j l}, \alpha_{j l}, \delta_{j l}\right\}$ for $j=1, \ldots, m$ can be updated by maximizing the observed log-likelihood function (using a Newton-Raphson algorithm):

$$
\mathcal{L}^{*}=c-\frac{1}{2} \sum_{t=1}^{n} \sum_{j=1}^{m} S_{t}(j)\left[\log \left|\boldsymbol{\Sigma}_{t / t-1}^{j}\right|+\left(\mathbf{y}_{t}-\theta_{j}\right)^{\prime} \boldsymbol{\Sigma}_{t / t-1}^{j-1}\left(\mathbf{y}_{t}-\theta_{j}\right)\right]
$$

\section{$5 \quad$ Experimental Results}

In this section, we study the performance of our proposed algorithm using synthetic and financial data.

\subsection{Model Learning and Stability of the Estimates}

The example used here has $q=6$ series, $m=3$ hidden states and only one common factor. The regime switching dates are $t_{1}^{*}=n / 3+1$ and $t_{2}^{*}=2 n / 3+$ 1. The iterations of the EM algorithm 2 stop when the relative change in the likelihood function between two subsequent iterations is smaller than a threshold value $=10^{-4}$. In this experiment we try to estimate the parameters of the model and to study the behavior of the estimates when the size of the sequence $n$ increases. A natural metric to measure the distance of estimators from the true parameters is the Kullback-Leibler divergence (Juang and Rabiner [5]). For each value of $n$, the estimation procedure was carried out a hundred times, and the distances $\widetilde{K}_{n}\left(\Theta_{0}, \widetilde{\Theta}_{n}\right)$ between each of the hundred estimators and the true parameter $\Theta_{0}$ were evaluated on a new sequence, independent of the first hundred sequences used to obtain the estimators. Figure 1 clearly shows a general decrease in average and spread of the distances with increasing $n$. This imply an increasing accuracy and stability of the estimators as $n$ increases.

\subsection{Model Selection Procedure}

Comparing the adequacy of different models may be done by computing a criterion for each model and comparing the criteria values. In this experiment BIC

$\overline{2}$ The initial parameters for the EM algorithm, were obtained by randomly perturbing the true parameter values by up to $20 \%$ of their true value. 


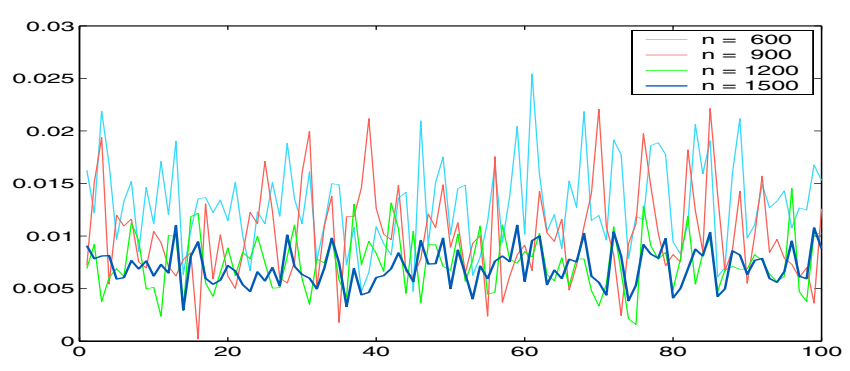

Fig. 1. Box plots of $\widetilde{K}\left(\Theta_{0}, \widetilde{\Theta}_{n}\right)$

and ICL criteria are used. The ICL criterion, is based on the maximization of the integrated complete log-likelihood function (Biernacki and Celeux [6]). Here we consider two different heteroskedastic specifications. For the two examples, $q=6$ and $n=900$ was used. In the first case the true model is the one used in 5.1. In the second case, we take $m=k=2$ and $t^{*}=n / 2+1$.

The steps for the model selection procedure are as follows. For each selection criterion, first, train various model configurations (obtained by varying the number of states and the number of factors). Second, use the output of EM to compute the values of the selection criterion for all configurations and select the one that yields the lowest value. In the two examples, random initialisation was used for the implementation of the learning algorithm. With this intention, we generated 100 different data experiments according to the true model. In the first case the results show that BIC and ICL chose 3 states and one factor most of the time $(68 \%, 73 \%)$. This is the best classification, since the use of one or two states is not enough to represent the data, and choosing two factors corresponds to an overfitting. In the second case, BIC and ICL choose also the true specification most of the time $(79 \%, 81 \%)$.

\subsection{Financial Data}

We have applied our model also to learn and analyze the co-movements amongst several exchange rate returns during the period where the European exchange rate mechanism has experienced a succession of crisis which reached its first culmination at the end of August 1992. What has been the impact of these changes on the nature of volatility? Has the degree of co-movement increased or decreased? Have common fluctuations become more or less volatile? Has the impact of crises on individual countries evolved over time? These questions are of interest to both policy makers and academic economists. For example, the questions of whether the common volatility has increased or declined, and whether countries have become more or less symmetric, are central to monetary and fiscal policy issues.

The time series considered here are the weekly average returns of closing spot prices relative to the US Dollar of the FRF, CHF, ITL, DEM, BEF, ESP, SEK, and GBP from 07/17/1985 to 01/22/1997 (600 observations). We trained 


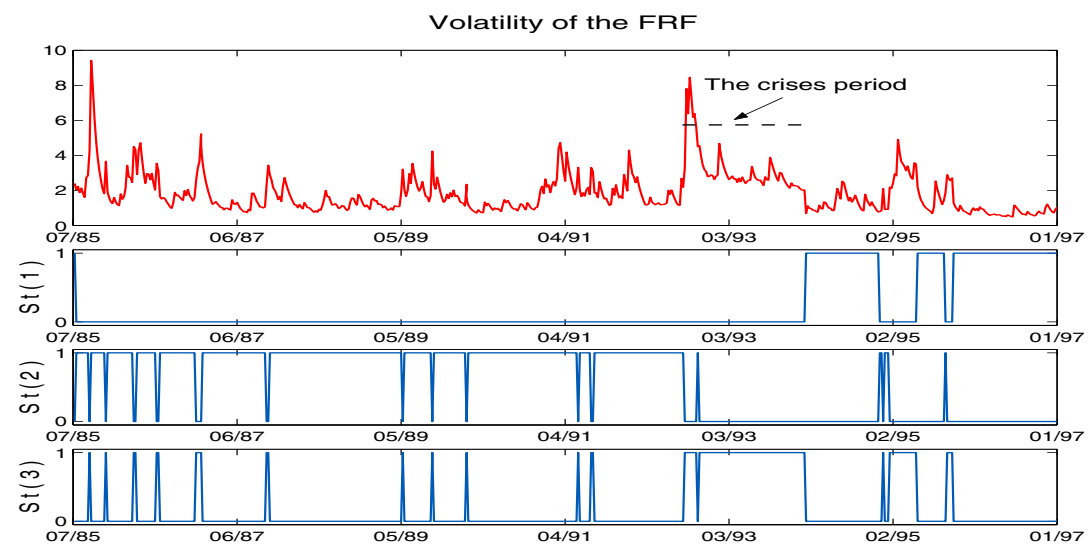

Fig. 2. Estimated Volatility of the French Franc (Graphic 1) and the Values of $S_{t}(j)$ given by the Viterbi algorithm (Graphics 2, 3, 4)

various model configurations (obtained also by varying the number of states and the number of factors). The key point for initialization consists in implementing a standard EM algorithm by supposing that the factors are homoskedastic 3 . Thereafter and given the output of the EM algorithm, we can use the values of $S_{t}(j)$ in order to obtain the optimal sequence of hidden states. At the second step, a particular simple conditionally heteroskedastic factor model is initialized for each segment. For this, one can use the empirical covariance matrices as estimates of the idiosyncratic variance matrices $\boldsymbol{\Psi}_{j}$ and the empirical means as estimates of the means $\theta_{j}$. The parameters of the conditionally heteroskedastic variances are initialized by applying a $\operatorname{GQARCH}(1,1)$ model to each segment of data. Finally, the elements of the transition matrix $\mathbf{P}$, can be initialized by counting the number of transitions from state $i$ to state $j$ and dividing by the number of transitions from state $i$ to any other state.

All the selection criteria argue that the time varying covariance structure could be modeled by two conditionally heteroskedastic common factors and three markovian regimes. For example in the cas of French Franc, figure 2 shows how the model is capable of accurately detecting abrupt changes in the time series structure and, in particular, the severe disruption by the violent storm which hit the European currency markets in September and October 1992. This segmentation shows that the third model is responsible for the high volatility segments, the second model is mainly responsible for the time period before September 1992, and the first for the lower volatility segments after 1993. This figure shows also that the average duration stay in the first regime is about 31.88 months versus 89.38 in the second and 28.73 in the third. For the two common factors, estimated $\alpha_{i}$ and $\delta_{i}$ are both statistically significant and their sum is slightly less than one which indicates strong GARCH effects and persistence in the volatility of exchange rates. The results show also that all the correlations between the

$\overline{3}$ In practice, 20 iterations of the EM algorithm are largely sufficient. 
different currencies have declined just after August 1992. This is not surprising because at the end of 1992 the range of the Exchange Rate Mechanism expanded to $30 \%$, which practically meant a return to free fluctuation.

\section{Conclusion}

The paper has developed a novel solution to the problem of modeling conditionally heteroskedastic financial time series subject to Markov switching within a multivariate framework. This new specification takes into account, simultaneously, the usual changing behavior of the common volatility due to common economic forces, as well as the sudden discrete shift in common and idiosyncratic volatilities that can be due to sudden abnormal events.

One of the most interesting applications of this new dynamic specification in finance is the possibility of obtaining on-line predictions of the time varying covariance matrices that is useful for dynamic asset allocation, active portfolio management and the analysis of options prices. The analysis in this paper can be also extended in several ways. First, our model can be generalized to one where one allows the idiosyncratic variances to be a stochastic function of time. Secondly, we can also think of the case where the state transition probabilities are not homogeneous in time, but depend on the previous state and the previously observed covariates levels. The study of such models would provide more flexibility in financial applications.

\section{References}

1. Engle R., Ng V.K., Rothschild M.: A Multi-Dynamic Factor Model for Stock Returns. J. of Econometrics. 52 (1992) 245-266

2. Diebold F., Nerlove M.: The Dynamics of Exchange Rate Volatility: A Multivariate Latent Factor ARCH Model. J. of Applied Econometrics. 4 (1988) 1-22

3. Engle R., Susmel R.: Common Volatility in International Equity Markets. J. of Business and Economic Statistics. 11 (1993) 369-380

4. Rosti A-V.I. Gales M.J.F.: Generalised Linear Gaussian Models. CUED/FINFENG/TR 420, Cambridge University (2001)

5. Juang B.H., Rabiner L.: Mixture autoregressive hidden Markov models for speech signals. IEEE Trans. on ASSP. 33 (1985) 1404-1413

6. Biernacki C., Celeux G., Assessing a mixture model for clustering with the integrated completed likelihood. IEEE Trans. on PAMI. 22 (2000) 719-725 